\title{
Wnt3a increases the metastatic potential of non-small cell lung cancer cells in vitro in part via its upregulation of Notch3
}

\author{
CHUNYAN LI ${ }^{1}$, GONGRU SONG $^{2}$, SIYANG ZHANG $^{1}$, ENHUA WANG $^{3}$ and ZESHI CUI ${ }^{1}$ \\ ${ }^{1}$ Center of Laboratory Technology and Experimental Medicine and Departments of ${ }^{2}$ Biotechnology, \\ ${ }^{3}$ Pathology, China Medical University, Shenyang, Liaoning 110001, P.R. China
}

Received October 24, 2014; Accepted December 11, 2014

DOI: $10.3892 /$ or.2014.3700

\begin{abstract}
Metastasis is the leading cause of death in lung cancer. Understanding the mechanisms underlying the process of metastasis is crucial for identifying novel anti-metastatic therapies. Studies indicate that the highly conserved developmental pathways, such as the Wnt and Notch signaling pathways, play important roles in the nonsmall cell lung cancer (NSCLC) tumorigenesis. However, the roles of both pathways in NSCLC metastasis are unclear. The present study aimed to investigate whether Wnt3a and Notch3, key components of the Wnt and Notch signaling pathways, respectively, regulate the metastatic abilities of NSCLC cells and whether there is some relationship during these regulatory events. Here, we observed that Wnt3a treatment upregulated, not only the protein expression of Notch3, but also the mRNA expression of Notch3 and its downstream genes, HES1 and HEYL. In addition, Wnt3a promoted cell invasion and anchorage-independent growth. Meanwhile, Wnt3a treatment caused epithelial-mesenchymal transition (EMT)-like morphological changes and F-actin reorganization. The western blotting data showed that Wnt3a treatment decreased the expression of E-cadherin and increased the expression of $\mathrm{N}$-cadherin and vimentin. Compared with Wnt3a treatment, Notch3 shRNA transfection had opposite effects. Furthermore, Notch3 shRNA weakened the effects of Wnt3a treatment on the in vitro cell invasion and EMT. Overall, these observations suggest that Wnt3a and Notch3 may promote the metastasis of NSCLC and Notch 3 upregulation is required for the Wnt3a mediated increased metastatic abilities of NSCLC.
\end{abstract}

Correspondence to: $\mathrm{Dr}$ Chunyan Li, Center of Laboratory Technology and Experimental Medicine, China Medical University, Shenyang, Liaoning 110001, P.R. China

E-mail: chyli@mail.cmu.edu.cn

Key words: Wnt3a, Notch3, non-small cell lung cancer cells, invasion, epithelial-mesenchymal transition

\section{Introduction}

Lung cancer still remains one of the most prevalent types of cancer and the leading cause of cancer-related deaths in the world. Metastasis is a common feature of lung cancer. Approximately $90 \%$ of lung cancer patient deaths are due to metastasis, rather than to the primary tumor (1). However, currently the processes and steps involved in metastasis are too complex to understand at a sufficient level. These facts highlight the need for investigating and clarifying the molecular mechanisms underlying and modulating lung cancer metastasis, in order to find novel therapeutic targets.

The aberrant activation of the Wnt and Notch signaling pathways, evolutionarily conserved pathways governing embryonic development, have been reported to contribute to lung cancer metastasis. Wnt ligands are a family of secreted glycoproteins. Through the binding of Wnt ligands to Frizzled and the low-density lipoprotein receptor-related protein-5/6 (LRP5/6) receptor, the canonical Wnt signaling pathway is initially activated and prevents the degradation of $\beta$-catenin in the cytoplasm causing it to accumulate in the nucleus and bind to the transcription factor of lymphoid enhancer-binding factor and the T-cell factor (LEF/TCF) to regulate expression of various genes. The aberrant activation of the Wnt signaling pathway promotes the colony formation and invasion of non-small cell lung cancer (NSCLC) cells, while antagonists to the Wnt signaling pathway inhibit epithelial-mesenchymal transition (EMT), cell migration and invasion (2-7). In mammals, the notch family consists of four Notch receptors (Notch1-4) and five ligands (Jagged1-2, delta-like 1, 3 and 4). Upon the interaction of notch ligands expressed on one cell with the receptors on an adjacent cell, the intracellular portion of the receptor is released and translocates to the nucleus where it interacts with recombining binding protein for immunoglobulin $\kappa \mathrm{J}$ region (RBP-J) and leads to the release and activation of the transcriptional co-repressors. Notch1-4 are upregulated in NSCLC tissues and Notch1 and 2 are positively correlated with lymph node metastasis (8). The inhibition of the Notch signaling pathway was found to decrease the invasive ability of NSCLC cells (9).

Although the role of the Wnt and Notch signaling pathways in NSCLC metastasis has been highlighted, it is not 
fully understood. In our previous study, we observed that the Wnt and Notch signaling pathways synergistically promoted NSCLC cell proliferation (10). As known, tumor metastasis is largely affected by cell proliferation in vivo $(11,12)$. This raises a question of whether there is some relationship between the Wnt and Notch signaling pathways in the modulation of metastasis. In the present study, we investigated the effects of Wnt3a, a Wnt signaling agonist, or Notch3 shRNA, or the combined application of Wnt3a and Notch3 shRNA on the metastatic abilities of NSCLC cells, such as cell invasion, anchorage-independent growth in soft agar and EMT in vitro.

\section{Materials and methods}

Cell culture and treatment. Three human lung cancer cell lines (A549, H157 and H460) were cultured in a DMEM/ F12 medium supplemented with $10 \%$ fetal calf serum (FCS) (both from HyClone, Beijing, China). Recombinant Wnt3a (5036-WNP-010; R\&D Systems, Minneapolis, MN, USA) was reconstituted at $200 \mu \mathrm{g} / \mathrm{ml}$ in sterile PBS containing at least $0.1 \%$ bovine serum albumin and stored at $-20^{\circ} \mathrm{C}$. The cells were placed in a fresh medium plus 50 or $100 \mathrm{ng} / \mathrm{ml} \mathrm{Wnt3a}$ and were cultivated for $24 \mathrm{~h}$ before cell analysis. PBS was used as control.

The plasmid encoding the shRNA targeting the Notch3 gene (sc-37136-SH; Santa Cruz Biotechnology, USA) and the non-target shRNA control plasmid were transfected into cells with TranSmarter (Abmart, Shanghai, China). After 48 h of transfection, the cells were selected with puromycin (Sigma-Aldrich, St. Louis, MO, USA) at a final concentration of $1 \mu \mathrm{g} / \mathrm{ml}$ for 2 weeks. Puromycin-resistant clones were isolated and subsequently cultured for the following experiment. Validation of the Notch 3 silencing in the transfected cells was carried out by western blotting.

Immunofluorescence. The cells seeded in 35-mm dishes were stained according to the following protocol. The medium mixture was discarded and 4\% paraformaldehyde was added to fix the cells at room temperature (RT) for $10 \mathrm{~min}$, and cells were washed with PBS for $5 \mathrm{~min}$ in a shaker and $0.2 \%$ Triton X-100 was added for $10 \mathrm{~min}$. The cells were washed with PBS twice and TRITC-conjugated phalloidin (Sigma-Aldrich) was added at RT for $40 \mathrm{~min}$, and the cells were washed with PBS containing $0.02 \%$ Triton X-100 for three times. Nuclei were counterstained with Hoechst $33342(5 \mu \mathrm{g} / \mathrm{ml})$ for $30 \mathrm{~min}$ at RT, cells were washed with PBS containing $0.02 \%$ Triton X-100 for three times and finally, $200 \mu \mathrm{l}$ PBS was added. The staining was examined under a laser scanning confocal microscope (FV1000; Olympus, Tokyo, Japan).

Reverse transcription (RT)-polymerase chain reaction $(P C R)$. The total RNA was prepared from the cultured cells using TRIzol reagent (Invitrogen Inc., Carlsbad, CA, USA) according to the manufacturer's instructions. The cDNA was synthesized using oligo(dT) as a primer. Primers (Table I) were designed with Primer3 on line. The PCR was carried out using a PCR kit (RT-PCR AMV 3.0 kit; Takara, Tokyo, Japan). The PCR products were electrophoresed on a $2 \%$ agarose gel and visualized by staining with GeneFinder (Baiweixin, Xiamen, China). The bands were quantified by densitometry to obtain the integrated density values (IDV). The relative amounts of each protein to glyceraldehyde-3-phosphate dehydrogenase (GAPDH) are represented as the ratio of their IDV in the histograms.

Cell invasion assay. The cells in the medium with $1 \%$ FCS were seeded into the upper chambers of a 24-well Transwell plate (3422; Corning, NY, USA) with BioCoat Matrigel (1:8; BD Bioscience, San Jose, CA, USA). The medium with $10 \%$ FCS was added to the lower chambers as a chemoattractant. After $24 \mathrm{~h}$ of incubation, cells that invaded through the membrane filter were fixed with $75 \%$ ethanol and stained with $0.1 \%$ crystal violet. The number of invading cells was counted under an inverted microscope with a digital CCD imaging system [IX70/SPOT RT-KE (color), Olympus/DI, Japan/USA] with a $\mathrm{X} 10$ objective in five random fields.

Anchorage-independent colony formation assay in soft agar. Soft agar plates were prepared using a Gene Med soft-agar kit (GMS10024; Gene Med, Shanghai, China) according to the manufacturer's instructions. To prepare the base layer, reagent A mixed with reagent $B$ in equal volumes was added into the 12-well plate and incubated at $50^{\circ} \mathrm{C}$ for $2 \mathrm{~h}$ to allow the agar to solidify. Top layer agar was prepared by mixing reagent $\mathrm{C}$ with reagent $A$ at a 3:1 ratio for semi-solidity, and the cells suspended in this top layer of agar were plated over the base layer. Then, the plates were incubated at $37^{\circ} \mathrm{C}$ overnight. The next day the liquid reagent $\mathrm{D}$ was added over the top layer of agar. Moreover, the cultures were fed with $0.25 \mathrm{ml}$ of reagent $\mathrm{D}$ at 2-day intervals for 2 weeks. In the Wnt3a treatment groups, Wnt3a was added to the semi-solidity agar reagent and the reagent $\mathrm{D}$ was replenished to the concentration of $100 \mathrm{ng} / \mathrm{ml}$. Finally, colonies with diameter $>120 \mu \mathrm{m}$ were scored under a light microscope at low magnification [IX70/SPOT RT-KE (color), Olympus/DI, Japan/USA]. The numbers of the colonies in the treatment groups were converted to a percentage of the control (by considering the control as 100\%).

Western blot analysis. Cells were lysed in a phospho-lysis buffer (50 mM Tris-Cl, pH 7.5, $150 \mathrm{mM} \mathrm{NaCl}, 1 \mathrm{mM} \mathrm{MgCl}{ }_{2}$, $0.5 \% \mathrm{NP}-40,1 \mathrm{mg} / \mathrm{ml}$ BSA, $0.1 \mathrm{mM}$ PMSF). Samples were analyzed by $10 \%$ SDS-polyacrylamide gel electrophoresis, followed by western blotting using rabbit monoclonal antiNotch3 (D11B8; Cell Signaling Technology, Beverly, MA, USA), rabbit polyclonal N-cadherin (W745), rabbit polyclonal E-cadherin (R868), (both from Bioworld Technology, Nanjing, China), and vimentin (ab8069; Abcam, Cambridge, MA, USA), respectively, and the secondary antibody of goat anti-rabbit IgG conjugated with horseradish peroxidase (HRP; HuaAn Biotech, Hangzhou, China). The membrane was developed using chemiluminescent reagents (SuperSignal West Pico Chemiluminescent Substrate; Pierce, Rockford, IL, USA). The bands were quantified by densitometry to obtain the IDV. The relative amounts of each protein to actin are represented as the ratio of their IDV in the histograms.

Statistical analysis. Data are presented as the mean \pm SE of at least three independent experiments. The one-way analysis of variance (ANOVA) was used for statistical analysis. Statistical significance was accepted at $\mathrm{P}<0.05$. 
A

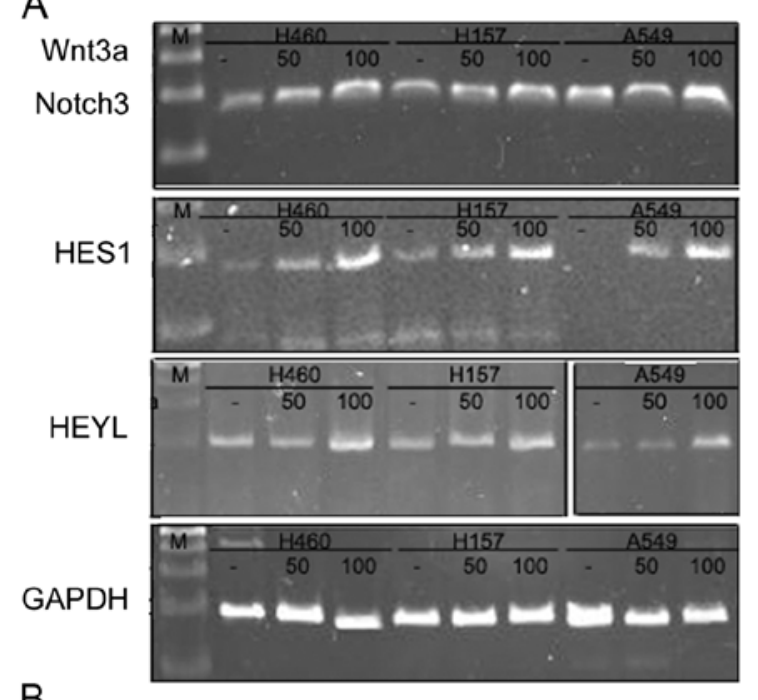

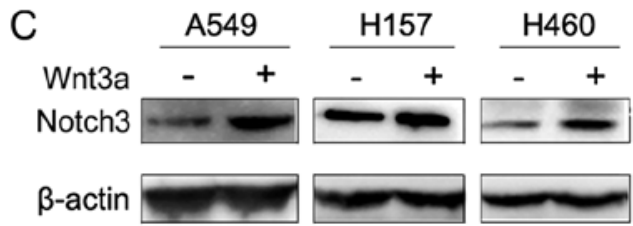

D

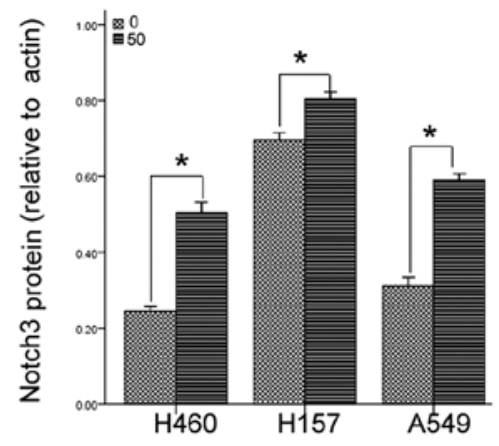

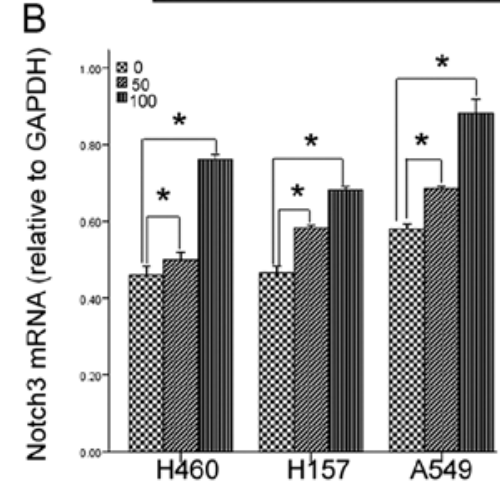
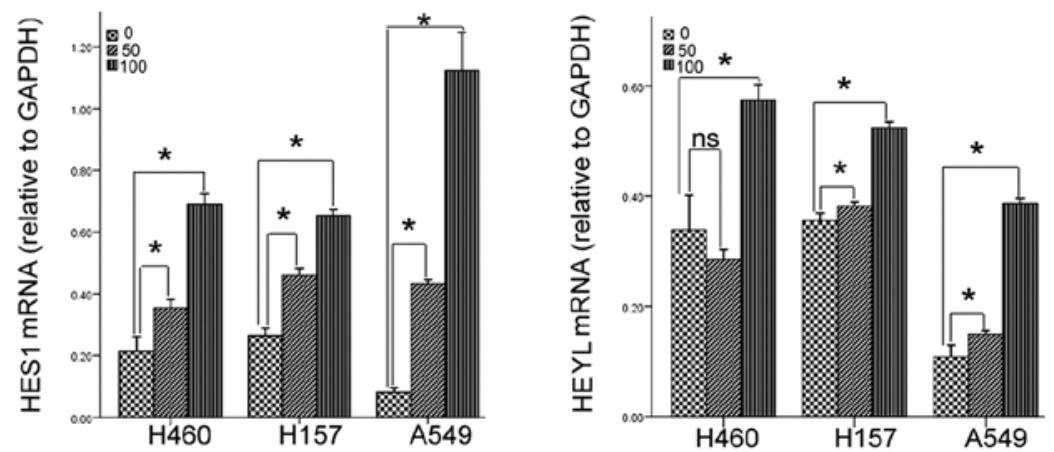

Figure 1. Wnt3a treatment upregulates the expression of Notch3 and its downstream genes. (A) PCR amplification of Notch3, HES1 and HEYL. The mRNA expression of Notch3, HES1 and HEYL in the NSCLC lines following Wnt3a treatment was higher than that in the control. (B) The histogram showing densitometric analysis represents the mRNA levels of Notch3, HES1 and HEYL as a ratio with respect to GAPDH levels. Wnt3a treatment significantly increased the mRNA expression of Notch3, HES1 and HEYL. The error bar represents mean \pm SE. (C) Western blotting panels of Notch3. The protein expression of Notch 3 following treatment with $100 \mathrm{ng} / \mathrm{ml} \mathrm{Wnt3a} \mathrm{was} \mathrm{higher} \mathrm{than} \mathrm{that} \mathrm{in} \mathrm{the} \mathrm{control.} \mathrm{(D)} \mathrm{The} \mathrm{histogram} \mathrm{of} \mathrm{densitometric} \mathrm{analysis} \mathrm{represents} \mathrm{the} \mathrm{protein} \mathrm{levels}$ of Notch3 as a ratio with respect to the actin levels. Wnt3a treatment significantly increased the protein expression of Notch3. The error bar represents mean \pm SE. GAPDH, glyceraldehyde-3-phosphate dehydrogenase. ${ }^{*} \mathrm{P}<0.05$ compared to the control. ns, non-significant.

Table I. Oligonucleotide primers used for RT-PCR analyses.

\begin{tabular}{llc}
\hline Gene & \multicolumn{1}{c}{ Primer sequence (strand) } & Product size (bp) \\
\hline Notch3 & 5'-caacccggtgtacgagaagt-3' (+) & 180 \\
& 5'-gaacgcagtagctcctctgg-3' (-) & \\
HES1 & 5'-ctctcttcctccggactct-3' (+) & 186 \\
& 5'-aggcgcaatccaatatgaac-3' (-) & \\
HEYL & 5'-caagcatgcaactccaaaga-3' (+) & 184 \\
& 5'-aggaaggcttggggatagaa-3' (-) & \\
GAPDH & 5'-tctgcccggagcctccttcc-3' (+) & 196 \\
& 5'-gatgcacccgctgcgcacta-3' (-) & \\
\hline
\end{tabular}

\section{Results}

Wnt3 a upregulates the expression of Notch3 and its down stream genes in NSCLC cell lines. Wnt3a, a member of the secreted Wnt ligands, is consistently used to mimic the biochemical effects of the canonical Wnt signaling pathway $(13,14)$. Notch3 is one of the four Notch receptors identified in mammals. Previous studies concerned with the pathogenesis of lung cancer have identified that Notch3 plays an essential role in NSCLC $(15,16)$. Compared with the control, Wnt3a treatment increased the mRNA expression of Notch 3 and its downstream gene, HES1, in all the three cell lines in a dose-dependent manner. As for HEYL, another downstream gene of Notch3, Wnt3a treatment dosedependently increased its mRNA expression in the H157 and A549 cells. In the $\mathrm{H} 460$ cells, only $100 \mathrm{ng} / \mathrm{ml}$ of Wnt3a increased the mRNA expression of HEYL (Fig. 1A and B). To further confirm the effects of Wnt3a on Notch3 expression, we assessed the protein level of Notch3, using western blotting in the absence or presence of $100 \mathrm{ng} / \mathrm{ml}$ of Wnt3a. Consistent with the data at the mRNA level, Wnt3a treatment significantly increased the expression of Notch3 at the protein level (Fig. 1C and D). Altogether, these data strongly indicate that Wnt3a may upregulate Notch3.

Notch3 shRNA specifically reduces Notch3 protein expression. Notch 3 is one of the four Notch receptors identified in mammals and its overexpression is frequently noted in NSCLC. In the present study, the expression of Notch3 was confirmed by western blotting. Three lung cancer cell lines 

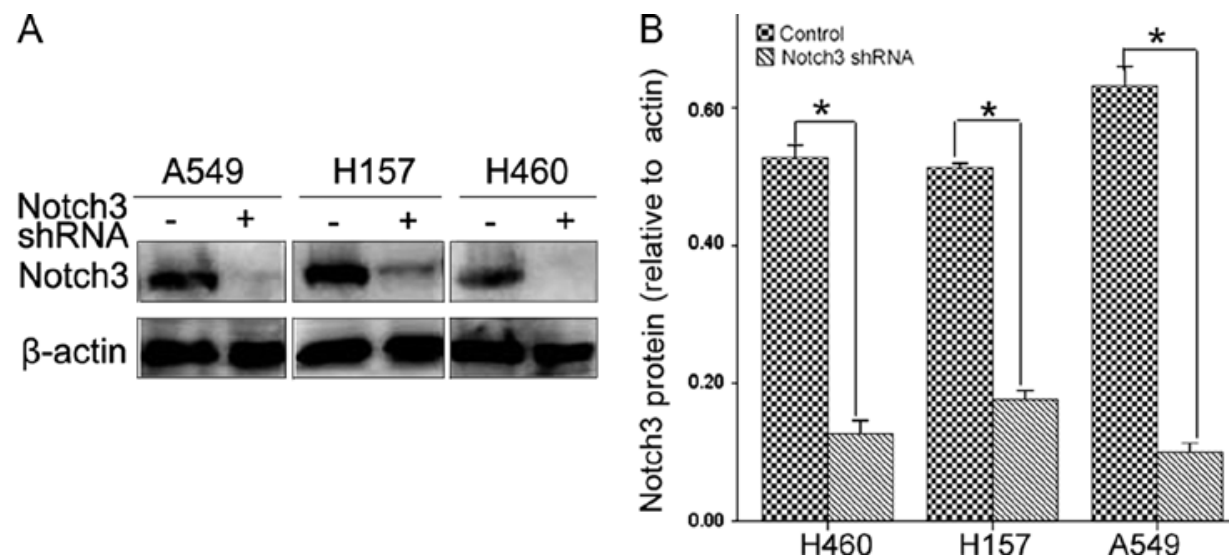

Figure 2. Notch3 shRNA significantly reduces protein expression. (A) The western blotting panels of Notch3. The protein expression of Notch3 in the Notch3 shRNA-transfected NSCLC cells was lower than that in the control. (B) The histogram of the densitometric analysis represents the protein levels of Notch 3 as a ratio with respect to the actin levels. The Notch3 shRNA significantly decreased the protein expression of Notch3. The error bar represents mean \pm SE. ${ }^{*} \mathrm{P}<0.05$ compared to the control.
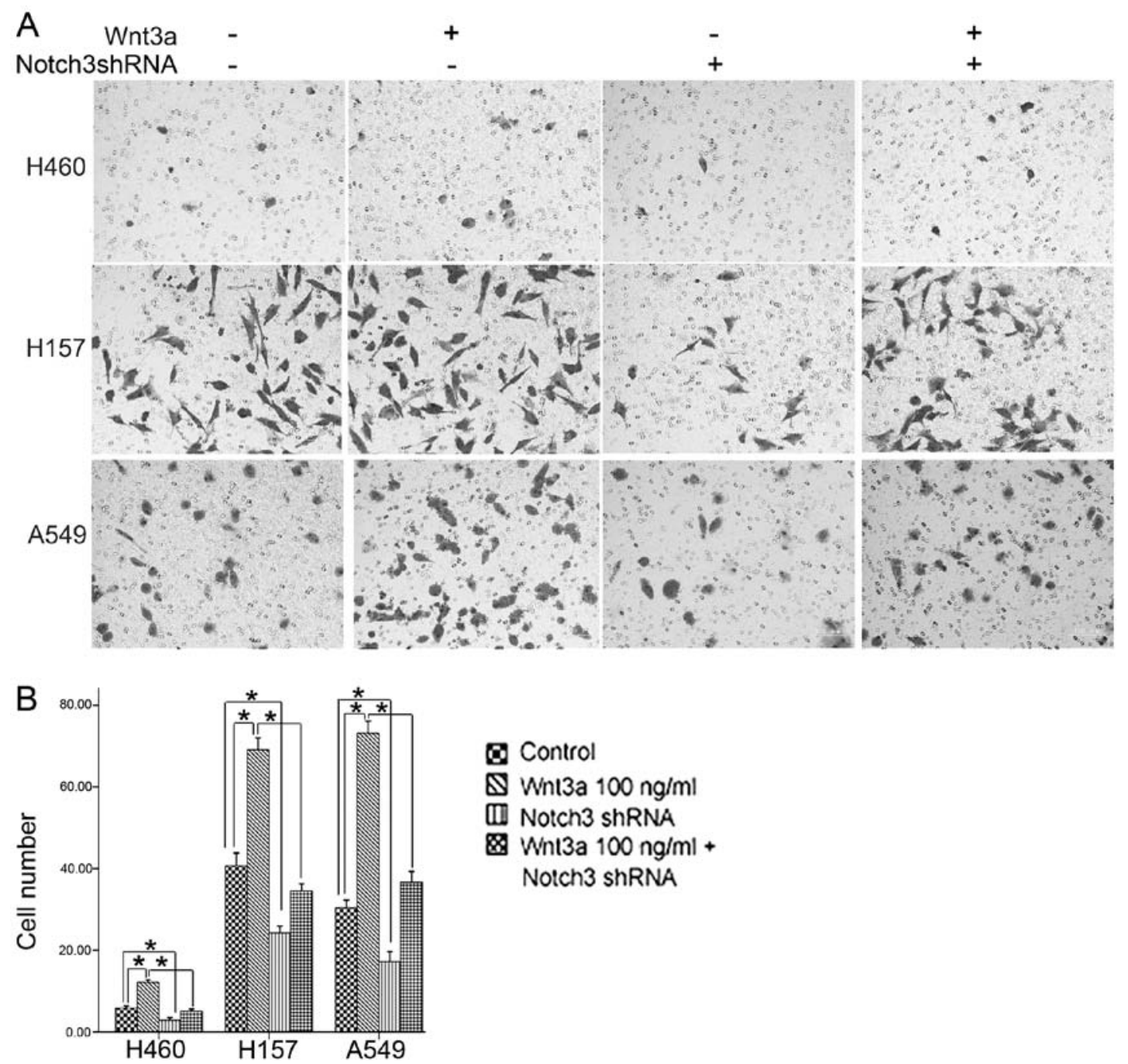

Figure 3. Roles of Wnt3a and Notch 3 in in vitro invasion. (A) Representative images of invading cells stained by crystal violet. Scale bars, $100 \mu \mathrm{m}$. (B) The histogram represents the cell numbers invading through the membrane. Wnt3a increased the number of invasive cells, whereas Notch 3 shRNA decreased the number of invasive cells. Notch3 shRNA efficiently decreased the effects of Wnt3a on the cell invasion in vitro. The error bar represents mean \pm SE. ${ }^{*} \mathrm{P}<0.05$ compared to the control.

transfected with Notch3 shRNA showed a significant reduction in Notch3 protein expression (Fig. 2A and B).
Roles of Wnt $3 a$ and Notch 3 in cell invasion. As in vitro invasion is one of the characteristics of metastasis, the Transwell 

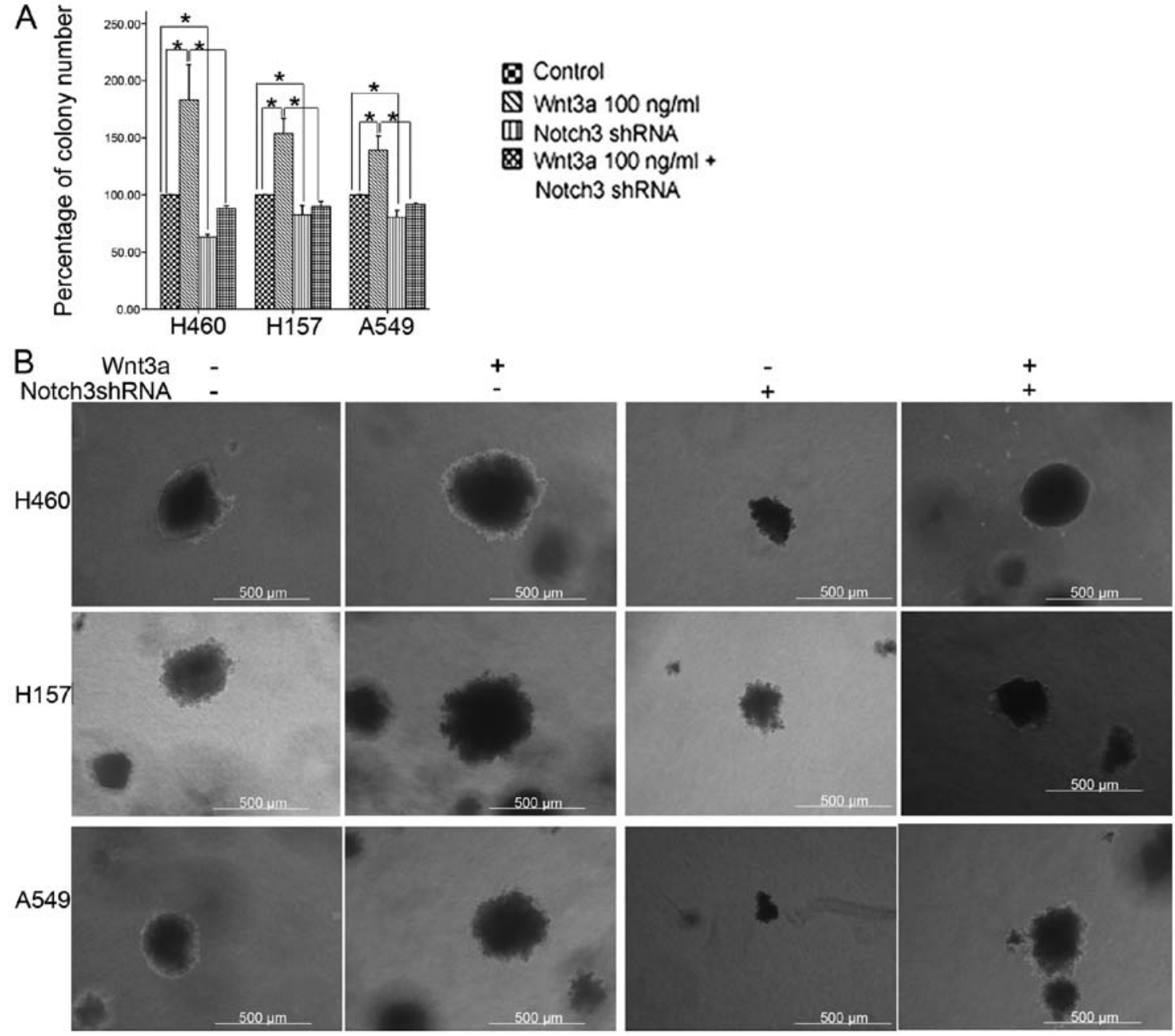

Figure 4. Roles of Wnt3a and Notch3 in the anchorage-independent growth in soft agar. (A) The histogram represents the colony numbers. The colony numbers were converted into a percentage of the control (control=100\%). Wnt3a increased the colony numbers, whereas Notch 3 shRNA decreased the colony numbers. Notch 3 shRNA efficiently decreased the effects of Wnt $3 a$ on the colony formation. The error bar represents mean $\pm \mathrm{SE}$. "P<0.05 compared to the control. (B) Representative images of the soft agar colony formation. The analysis demonstrated that the cells used in this experiment all formed distinctly visible colonies embedded in different layers of soft agar. Wnt3a treatment increased the sizes of the colonies. A reduction in Notch 3 decreased the sizes of the colonies. Scale bars, $500 \mu \mathrm{m}$.

invasion assay was carried out to assess the effects of Wnt3a and Notch 3 on cell invasion. Cell invasion was increased in the three cell lines treated with $100 \mathrm{ng} / \mathrm{ml} \mathrm{Wnt3a.} \mathrm{Notch} 3$ reduction led to decreased cell invasion in the three cell lines. Moreover, Notch3 reduction significantly attenuated the effects of Wnt3a treatment on the invasive ability of cells (Fig. 3A and B). The data demonstrated that Wnt3a and Notch 3 promoted in vitro invasion of the NSCLC cells and indicate that the Wnt pathway may promote cell invasion partially via Notch3.

Roles of Wnt3a and Notch 3 in the anchorage-independent growth in soft agar. For cells to undergo metastasis, they must have the ability to overcome anoikis and survive without cell-substratum interaction (17). The anchorage-independent growth in soft agar has been shown to be one of the independent factors of the metastatic potential in cancer $(18,19)$. To further analyze the effects of Wnt3a and Notch3 on the characteristics of metastatic outgrowth, a soft-agar colony assay was used in the present study. When cultured in the reagent with $100 \mathrm{ng} / \mathrm{ml}$ Wnt3a, the cells formed compact spherical colonies that grew larger in size and in number, when compared to the control cells. Meanwhile, Notch3 reduction decreased the size and the number of the colonies. Consistent with the results of the cell invasion assay, Notch3 reduction also weakened the effects of Wnt3a on the size and number of colonies, suggesting that Notch3 may be involved in the colony outgrowth prompted by the Wnt signaling pathway in the NSCLC cells (Fig. 4).

Roles of Wnt3a and Notch3 in the regulation of EMT. Accumulating evidence suggests that the EMT is a key event in cancer metastasis. During EMT, cell morphological changes and dynamic remodeling of the actin cytoskeleton consistently occur $(20,21)$. In the control cells, the majority of cells maintained typical epithelial morphology, while some of the Wnt3a-treated cells underwent elongation to become fibroblast-like spindle-shaped cells (Fig. 5A, black arrowhead). The F-actin staining also showed that some of the Wnt3a-treated cells became longer (Fig. 5B, white arrowhead). Moreover, we observed that the F-actin filaments of the control cells were mainly organized in cortical thin bundles whereas the actin 

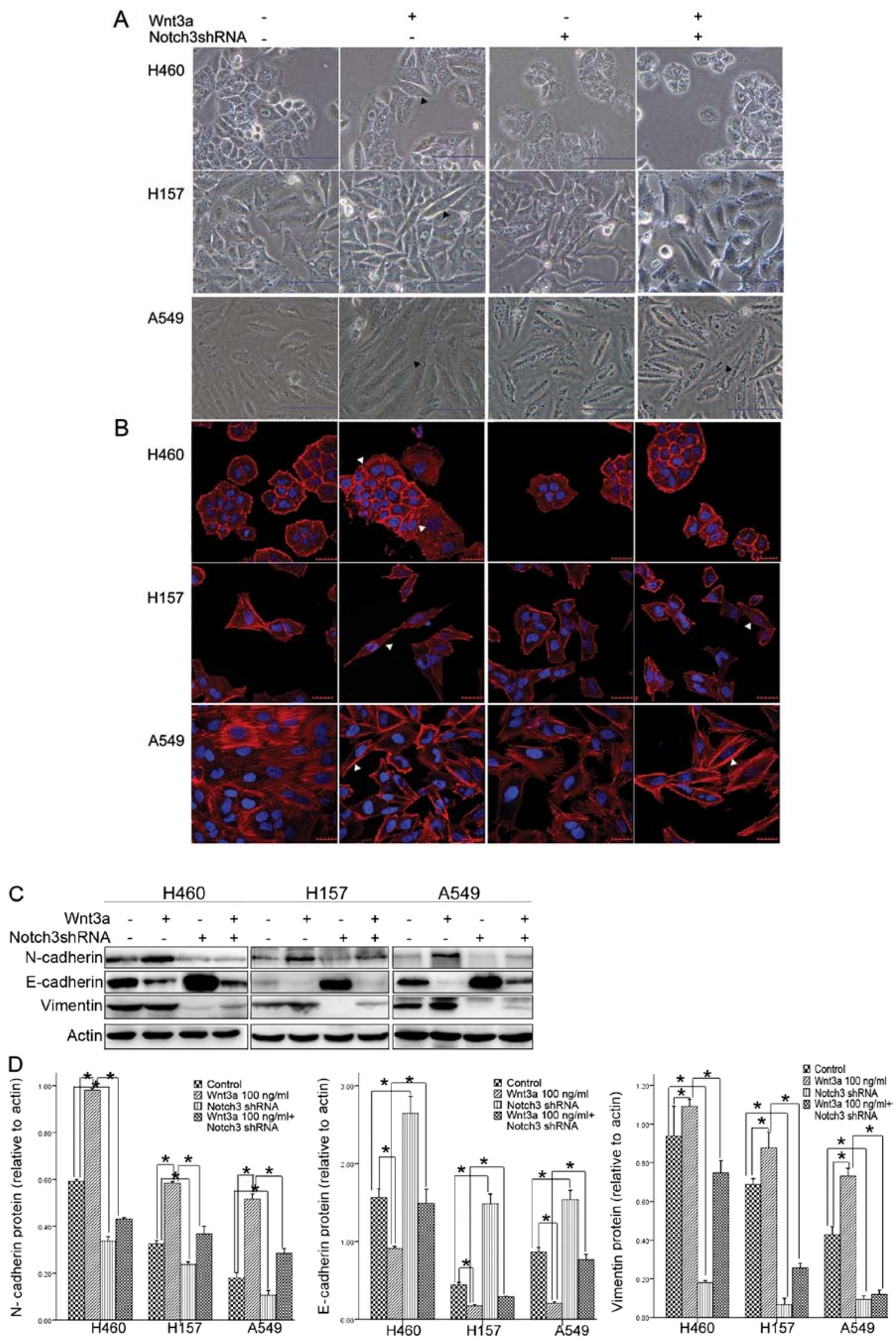

Figure 5. Roles of Wnt3a and Notch3 in the regulation of EMT. (A) Representative phase images of cell morphology. The control cells were typically epithelial. The black arrowheads show that some of the Wnt3a-treated cells underwent mesenchymal elongation. Scale bars, $100 \mu \mathrm{m}$. (B) Representative images of actin staining (actin is red and nucleus is blue). The white arrowheads indicate the stress fibers, the actin filaments and the elongated cells, respectively. Scale bar, $20 \mu \mathrm{m}$. (C) The western blotting panels of E-cadherin, N-cadherin and vimentin. (D) The histograms of the densitometric analysis represent the protein levels of E-cadherin, $\mathrm{N}$-cadherin and vimentin as a ratio to the respective actin levels. Wnt3a increased the expression of $\mathrm{N}$-cadherin and vimentin and decreased the expression of E-cadherin. Notch3 shRNA showed the opposite effects and efficiently weakened the effects of Wnt3a on the expression of these markers. "P<0.05 compared to the control. EMT, epithelial-mesenchymal transition. 
filaments of the Wnt3a-treated cells appeared to be assembled into thick bundles at the cell surface in the A549 cells (Fig. 5B, white arrowhead). However, compared to the Wnt3a treatment, Notch3 shRNA appeared to have opposite effects on the cell morphology and actin reorganization (Fig. 5A and B). These results indicate that Wnt3a and Notch3 may induce the EMT process in the cultured cells.

To further explore the roles of Wnt3a and Notch3 on EMT, we measured the protein expression levels of E-cadherin, $\mathrm{N}$-cadherin and vimentin, key protein markers involved in EMT. The downregulation of the epithelial marker E-cadherin and the upregulation of the mesenchymal markers $\mathrm{N}$-cadherin and vimentin are all hallmarks of EMT (20). Wnt3a treatment significantly induced the downregulation of E-cadherin and the upregulation of $\mathrm{N}$-cadherin and vimentin at the protein level. In contrast, Notch3 shRNA significantly induced the downregulation of $\mathrm{N}$-cadherin and vimentin and the upregulation of E-cadherin. Moreover, Notch3 shRNA partly inhibited the regulation of $\mathrm{Wnt} 3 \mathrm{a}$ on the expression of three protein factors (Fig. 5C and D). Taken together, these findings indicate that Wnt3a treatment is sufficient to induce EMT in the NSCLC cells, in which Notch3 is involved.

\section{Discussion}

Metastasis occurs as a multi-step process during which cancer cells in the primary tumor lose cell-cell adhesion and break through the basement membrane with increased invasive properties and intravasate the circulation to be transported to distant tissues, extravasate out of the circulatory system and subsequently colonize remote sites $(22,23)$. Both the Wnt and Notch signaling pathways have been reported to transform cancer cells to an invasive and metastatic phenotype. The canonical Wnt signaling pathway may contribute to the metastatic progression of cancer by promoting EMT, regulating the expression of matrix metalloproteinases (MMPs) and other factors that play a role in the regulation of the extracellular matrix (24-28). The notch signaling pathway may participate in cancer metastasis by the establishment of stem cell populations that allow for the creation of metastatic niches and its interaction with hypoxia can accelerate the steps of invasion and metastasis in cancer modulating EMT (29-31).

Wnt3a and Notch3 are important components of the Wnt and Notch signaling pathways, respectively. In the present study, Wnt3a-treated cells exhibited increased invasion ability and anchorage-independent growth compared to the controls. Moreover, Wnt3a introduced the EMT-like elongation of cell morphology, F-actin reorganization as well as the downregulation of E-cadherin and upregulation of $\mathrm{N}$-cadherin and vimentin. In contrast, Notch3 shRNA transfection had the opposite effects on the invasion ability, the anchorageindependent growth cell morphology, F-actin reorganization and the expression of EMT markers, compared with Wnt3a treatment. These observations are consistent with previous studies that Wnt3a and Notch3 induce EMT and promote metastasis in other types of cancer (32-34). Therefore, the data of the present study strongly indicate that both Wnt3a and Notch3 may positively regulate NSCLC metastasis.

The Wnt and Notch signaling pathways are closely interconnected in the development of various types of cancer and their interaction is indispensable for tumorigenesis, such as breast cancer or colon cancer $(35,36)$. However, the crosstalk between the Wnt and Notch signaling pathways in NSCLC metastasis is poorly addressed. In our experiment, Wnt3a upregulated the expression of Notch 3 and downstream genes. Moreover, Wnt3a induced EMT and the in vitro characteristics of metastasis were partially reversed by the knockdown of Notch3. Together, these findings indicate that the crosstalk between the Wnt and the Notch signaling pathways may exist in NSCLC metastasis.

Although further studies are still required to clarify how Wnt3a and Notch 3 cooperatively promote NSCLC metastasis, our findings may support the existence of crosstalk between the two pathways during the metastatic process in NSCLC cells. The results of the present study may facilitate our understanding of the molecular mechanisms modulating NSCLC metastasis. This should be important for the identification of novel targets to prevent NSCLC progression.

\section{Acknowledgements}

This research was sponsored by the Scientific Research Foundation for the Returned Overseas Chinese Scholars, State Education Ministry (to C.L., no. 20091001) and the Liaoning Nature Science Project (to C.L., no. 201102258).

\section{References}

1. Perlikos F, Harrington KJ and Syrigos KN: Key molecular mechanisms in lung cancer invasion and metastasis: A comprehensive review. Crit Rev Oncol Hematol 87: 1-11, 2013.

2. Xie C, Jiang G, Fan C, et al: ARMC8 $\alpha$ promotes proliferation and invasion of non-small cell lung cancer cells by activating the canonical Wnt signaling pathway. Tumour Biol 35: 8903-8911, 2014.

3. Gao Y, Song C, Hui L, et al: Overexpression of RNF146 in non-small cell lung cancer enhances proliferation and invasion of tumors through the Wnt $/ \beta$-catenin signaling pathway. PLoS One 9: e85377, 2014.

4. Su K, Huang L, Li W, et al: TC-1 (c8orf4) enhances aggressive biologic behavior in lung cancer through the $\mathrm{Wnt} / \beta$-catenin pathway. J Surg Res 185: 255-263, 2013.

5. Zhang S, Wang Y, Dai SD and Wang EH: Down-regulation of NKD1 increases the invasive potential of non-small-cell lung cancer and correlates with a poor prognosis. BMC Cancer 11: 186,2011

6. Ren J, Wang R, Huang G, Song H, Chen Y and Chen L: sFRP1 inhibits epithelial-mesenchymal transition in A549 human lung adenocarcinoma cell line. Cancer Biother Radiopharm 28: 565-571, 2013.

7. Singh T and Katiyar SK: Honokiol inhibits non-small cell lung cancer cell migration by targeting $\mathrm{PGE}_{2}$-mediated activation of $\beta$-catenin signaling. PLoS One 8: e60749, 2013.

8. Westhoff B, Colaluca IN, D'Ario G, et al: Alterations of the Notch pathway in lung cancer. Proc Natl Acad Sci USA 106: 22293-22298, 2009.

9. Liu L, Chen X, Wang Y, et al: Notch3 is important for TGF- $\beta$-induced epithelial-mesenchymal transition in non-small cell lung cancer bone metastasis by regulating ZEB-1. Cancer Gene Ther 21: 364-372, 2014

10. Li C, Zhang S, Lu Y, Zhang Y, Wang E and Cui Z: The roles of Notch 3 on the cell proliferation and apoptosis induced by CHIR99021 in NSCLC cell lines: a functional link between Wnt and Notch signaling pathways. PLoS One 8: e84659, 2013.

11. Gao CF, Xie Q, Su YL, et al: Proliferation and invasion: plasticity in tumor cells. Proc Natl Acad Sci USA 102: 10528-10533, 2005.

12. Al-Mehdi AB, Tozawa K, Fisher AB, Shientag L, Lee A and Muschel RJ: Intravascular origin of metastasis from the proliferation of endothelium-attached tumor cells: a new model for metastasis. Nat Med 6: 100-102, 2000. 
13. Kikuchi A, Yamamoto $\mathrm{H}$ and Kishida S: Multiplicity of the interactions of Wnt proteins and their receptors. Cell Signal 19: 659-671, 2007.

14. Stapp AD, Gómez BI, Gifford CA, Hallford DM and Hernandez Gifford JA: Canonical WNT signaling inhibits follicle stimulating hormone mediated steroidogenesis in primary cultures of rat granulosa cells. PLoS One 9: e86432, 2014.

15. Yi F, Amarasinghe B and Dang TP: Manic fringe inhibits tumor growth by suppressing Notch3 degradation in lung cancer. Am J Cancer Res 3: 490-499, 2013.

16. Zheng Y, de la Cruz CC, Sayles LC, et al: A rare population of CD24(+)ITGB4(+)Notch(hi) cells drives tumor propagation in NSCLC and requires Notch3 for self-renewal. Cancer Cell 24 59-74, 2013.

17. Frisch SM and Francis H: Disruption of epithelial cell-matrix interactions induces apoptosis. J Cell Biol1 24: 619-626, 1994.

18. Nomura Y, Tashiro $\mathrm{H}$ and Hisamatsu K: In vitro clonogenic growth and metastatic potential of human operable breast cancer. Cancer Res 49: 5288-5293, 1989.

19. Nakanishi K, Sakamoto M, Yasuda J, et al: Critical involvement of the phosphatidylinositol 3-kinase/Akt pathway in anchorage-independent growth and hematogeneous intrahepatic metastasis of liver cancer. Cancer Res 62: 2971-2975, 2002.

20. Tsai JH and Yang J: Epithelial-mesenchymal plasticity in carcinoma metastasis. Genes Dev 27: 2192-2206, 2013.

21. Huber MA, Kraut N and Beug H: Molecular requirements for epithelial-mesenchymal transition during tumor progression. Curr Opin Cell Biol 17: 548-558, 2005.

22. Gupta GP and Massagué J: Cancer metastasis: building a framework. Cell 127: 679-695, 2006.

23. Poste G and Fidler IJ: The pathogenesis of cancer metastasis. Nature 283: 139-146, 1980.

24. Bo H, Zhang S, Gao L, Chen Y, Zhang J, Chang X and Zhu M: Upregulation of Wnt5a promotes epithelial-to-mesenchymal transition and metastasis of pancreatic cancer cells. BMC Cancer 13: 496, 2013.

25. Ford CE, Jary E, Ma SS, Nixdorf S, Heinzelmann-Schwarz VA and Ward RL: The Wnt gatekeeper SFRP4 modulates EMT, cell migration and downstream Wnt signalling in serous ovarian cancer cells. PLoS One 8: e54362, 2013.
26. Kwon M, Lee SJ, Wang Y, et al: Filamin A interacting protein 1-like inhibits WNT signaling and MMP expression to suppress cancer cell invasion and metastasis. Int J Cancer 135: 48-60, 2014.

27. Jiang W, Crossman DK, Mitchell EH, Sohn P, Crowley MR and Serra R: WNT5A inhibits metastasis and alters splicing of Cd44 in breast cancer cells. PLoS One 8: e58329, 2013.

28. Dey N, Barwick BG, Moreno CS, et al: Wnt signaling in triple negative breast cancer is associated with metastasis. BMC Cancer 13: 537, 2013.

29. Espinoza I and Miele L: Deadly crosstalk: Notch signaling at the intersection of EMT and cancer stem cells. Cancer Lett 341: 41-45, 2013.

30. Espinoza I, Pochampally R, Xing F, Watabe K and Miele L: Notch signaling: targeting cancer stem cells and epithelial-to-mesenchymal transition. Onco Targets Ther 6: 1249-1259, 2013.

31. Du R, Sun W, Xia L, et al: Hypoxia-induced down-regulation of microRNA-34a promotes EMT by targeting the Notch signaling pathway in tubular epithelial cells. PLoS One 7: e30771, 2012.

32. Zhang Q, Bai X, Chen W, et al: Wnt/ $\beta$-catenin signaling enhances hypoxia-induced epithelial-mesenchymal transition in hepatocellular carcinoma via crosstalk with hif-1 $\alpha$ signaling. Carcinogenesis 34: 962-973, 2013.

33. Zhai Y, Iura A, Yeasmin S, et al: MSX2 is an oncogenic downstream target of activated WNT signaling in ovarian endometrioid adenocarcinoma. Oncogene 30: 4152-4162, 2011.

34. Gupta N, Xu Z, El-Sehemy A, Steed H and Fu Y: Notch3 induces epithelial-mesenchymal transition and attenuates carboplatin-induced apoptosis in ovarian cancer cells. Gynecol Oncol 130: 200-206, 2013.

35. Katoh M and Katoh M: NUMB is a break of WNT-Notch signaling cycle. Int J Mol Med 18: 517-521, 2006.

36. Bertrand FE, Angus CW, Partis WJ and Sigounas G: Developmental pathways in colon cancer: crosstalk between WNT, BMP, Hedgehog and Notch. Cell Cycle 11: 4344-4351, 2012. 